Research Article

\title{
Development, Optimization, and Evaluation of Luliconazole Nanoemulgel for the Treatment of Fungal Infection
}

\author{
Nabil A. Alhakamy, ${ }^{1,2,3}$ Shadab Md ${ }^{10},{ }^{1,2,3}$ Md Shoaib Alam, ${ }^{4}$ Rasheed A. Shaik $\left(D,{ }^{5}\right.$ \\ Javed Ahmad, ${ }^{6}$ Abrar Ahmad $\left(\mathbb{D},{ }^{7}\right.$ Hussam I. Kutbi, ${ }^{8}$ Ahmad O. Noor, ${ }^{8}$ Alaa Bagalagel, ${ }^{8}$ \\ Douha F. Bannan, ${ }^{8}$ Bapi Gorain,, ${ }^{9,}$ and Ponnurengam Malliappan Sivakumar $\mathbb{D}^{11}$ \\ ${ }^{1}$ Department of Pharmaceutics, Faculty of Pharmacy, King Abdulaziz University, Jeddah 21589, Saudi Arabia \\ ${ }^{2}$ Center of Excellence for Drug Research \& Pharmaceutical Industries, King Abdulaziz University, Jeddah 21589, Saudi Arabia \\ ${ }^{3}$ Mohamed Saeed Tamer Chair for Pharmaceutical Industries, King Abdulaziz University, Jeddah 21589, Saudi Arabia \\ ${ }^{4}$ Research \& Development, Jamjoom Pharmaceuticals, Jeddah 21442, Saudi Arabia \\ ${ }^{5}$ Department of Pharmacology \& Toxicology, Faculty of Pharmacy, King Abdulaziz University, Jeddah 21589, Saudi Arabia \\ ${ }^{6}$ Department of Pharmaceutics, College of Pharmacy, Najran University, Najran, Saudi Arabia \\ ${ }^{7}$ Department of Biochemistry, Faculty of Sciences, King Abdulaziz University, Jeddah 21589, Saudi Arabia \\ ${ }^{8}$ Department of Pharmacy Practice, Faculty of Pharmacy, King Abdulaziz University, Jeddah, Saudi Arabia \\ ${ }^{9}$ School of Pharmacy, Faculty of Health and Medical Sciences, Taylor's University, Subang Jaya 47500, Selangor, Malaysia \\ ${ }^{10}$ Centre for Drug Delivery and Molecular Pharmacology, Faculty of Health and Medical Sciences, Taylor's University, \\ Subang Jaya, Selangor, Malaysia \\ ${ }^{11}$ Sabanci University Nanotechnology Research and Application Center (SUNUM), Tuzla 34956, Istanbul, Turkey
}

Correspondence should be addressed to Shadab Md; shadabmd1982@gmail.com and Ponnurengam Malliappan Sivakumar; sivamedchem@gmail.com

Received 6 June 2021; Accepted 16 October 2021; Published 5 November 2021

Academic Editor: Bijan Das

Copyright (c) 2021 Nabil A. Alhakamy et al. This is an open access article distributed under the Creative Commons Attribution License, which permits unrestricted use, distribution, and reproduction in any medium, provided the original work is properly cited.

The present study aimed to optimize luliconazole nanoemulsion using Box-Behnken statistical design, which was further incorporated into the polymeric gel of Carbopol 934. The formulation was characterized for its size, entrapment efficiency, ex vivo permeation, and mechanism of release. The size of the dispersed globules of the optimized drug-loaded nanoemulsion was found to be $17 \pm 3.67 \mathrm{~nm}$ with a polydispersity index (PDI) less than 0.5 . Although the surface charge was recorded at $-9.53 \pm 0.251$, the stability was maintained by the polymeric matrix that prevented aggregation and coalescence of the dispersed globules. The luliconazole-nanoemulgel (LUL-NEG) was characterized for drug content analysis, viscosity, $\mathrm{pH}$, and refractive index, where the results were found to be $99.06 \pm 0.59 \%, 9.26 \pm 0.08$ Pa.s, $5.65 \pm 0.17$, and $1.31 \pm 0.08$, respectively. The permeation across the rat skin was found to be significantly higher with LUL-NEG when compared with LUL gel. Furthermore, the skin irritation test performed in experimental animals revealed that the blank NEG, as well as the LUL-NEG, did not produce any signs of erythema following $48 \mathrm{~h}$ exposure. In addition, the histopathological findings of the experimental skins reported no abnormal signs at the formulation application site. Finally, the NEG formulation was found to create a statistically significant zone of inhibition $(P<0.05)$ when compared to all other test groups. Overall, it could be summarized that the nanoemulgel approach of delivering luliconazole across the skin to treat skin fungal infections could be a promising strategy.

\section{Introduction}

Luliconazole (LUL), an antifungal drug containing imidazole moiety with ketone dithioacetate, is a broad-spectrum agent, which has shown its potential against wide varieties of fungi, especially against filamentous fungi, for example, dermatophytes [1]. Although the exact mechanism of this novel agent for antifungal efficacy is unknown, it has been reported that LUL acts by inhibiting the fungal cytochrome $\mathrm{P} 450$; that is, $14-\alpha$ demethylase enzyme thus prevents the 
biosynthesis of ergosterol from lanosterol and interrupts cell wall synthesis within the fungi [2-4]. Since 2013, the United States Food and Drug Administration has approved the commercial cream formulation of LUL $(1 \%, w / w)$ for clinical use against fungal infections and dermatophytosis including tinea corporis, tinea cruris, and tinea pedis resulting from the invasion of Epidermophyton floccosum and Trichophyton rubrum [5]. The solubility of LUL is low. This low solubility restricts permeation of the drug across the skin upon topical delivery [6]. Alternatively, conventional topical cream formulations possess several drawbacks of low permeation from the stratum corneum along with reduced retention at the site of application [7]. Moreover, the rate-limiting step for LUL permeation is its solubility in the lipid phase of the stratum corneum that limits its dermal availability [6]. With no formulations in the market other than cream and lotion for LUL, there is an urgent requirement for novel deliveries for improved retention and penetration from the site of skin application. A few research outcomes are available in the literature where different researchers tried to improve the delivery approach of LUL via liposomal and ethosomal gel preparation [7], lyotropic liquid crystalline nanoparticle [8], nanocrystals-loaded hydrogel [6], niosomal gel [9], solid lipid nanoparticle gel [10], and so on. The ethosomal and elastic liposomal-based gel preparation of LUL had reported safety and efficacy of the formulation without delivering the drug to the systemic circulation [7]. Similarly, $181 \pm 12.3 \mathrm{~nm}$ particle size of the LUL lyotropic liquid crystalline nanoparticle increased retention of the drug (LUL) on the stratum corneum and epidermis when compared with the commercial topical formulation. This increased retention time allowed the formulation to penetrate higher in the different layers of the skin [8]. An alternate formulation approach by Kumar and team depicted that nanocrystals-loaded hydrogel of LUL could retain highest in different skin layers when compared to the coarse suspension, nanosuspension, and D-gel [6]. Although extensive research was not conducted, it was concluded by Garg and team that the niosomal gel formulation of LUL could provide a platform for topical delivery against Candida [9]. Alternatively, topical gel delivery of LUL solid lipid nanoparticle showcased safety as there were no signs of oedema and erythema [10]. The advantages of different formulation approach on topical delivery of LUL have been implemented in this research via the development of nanoemulgel (NEG) delivery approach.

Advancement of NEG-based researches in the recent era has gained tremendous attention because of their stability, appearance, penetrability across the biological membranes, longer retention, and sustained release profile of the entrapped drug [11-14]. NEG is a novel biphasic polymeric nanoemulsion platform to topically deliver lipophilic agents, where nanometric size range of the oil globules facilitates permeation across the stratum corneum of the skin [11]. The polymeric networks in this architecture allow entrapping the dispersed globules promoting the stability of the formulation following incorporation of lipidic agents within the oil core of oil-in-water nanoemulsion. This NEG platform possesses the potential of enhanced solubility of lipophilic drugs, sustained release of the entrapped therapeutic agents, superior topical applicability, biocompatibility, and biodegradability $[15,16]$. The thixotropic NEG allows easy spreadability at the desired site and prolongs retention at the application site due to mucoadhesive property, where the hydrophilic nature of the formulation allows easy removal from the application site after accomplishment of desired efficacy [16, 17]. A wide number of researches have been conducted with NEG-based topical formulations of lipophilic drugs for improved efficacy of the entrapped therapeutics $[15,18,19]$.

Thus, the present study was attempted to develop and optimize LUL-loaded nanoemulgel for improved efficacy against fungal infection by enhancing the solubility and simultaneously the permeability across the skin barrier. Eucalyptus oil was selected as the lipid phase in the preparation of the nanoemulsion containing LUL for our study. This eucalyptus oil has been well documented for its antifungal efficacy $[20,21]$. Thus, it is hypothesized that the final NEG formulation of LUL would provide improved antifungal efficacy. The development of nanoemulsion was optimized using Box-Behnken statistical design and characterized. Furthermore, the permeation across the rat skin, zone of inhibition against Candida, and the skin irritability tests were performed to establish the superiority of the LULNEG over commercial preparation.

\section{Materials and Methods}

2.1. Materials. Luliconazole (purity $>98 \%$ ) was provided as a gift sample from Jamjoom Pharmaceutical, Jeddah, Saudi Arabia, whereas eucalyptus oil was obtained from Allin Exporters, Noida, India. Carbopol 934, Tween 20, and polyethylene glycol 200 (PEG 200) were purchased from Sigma Aldrich, MO, USA. The HPLC grade solvents were purchased from Merck, NJ, USA. The rest of the chemicals used in this current experiment were of analytical grade.

2.2. Preparation of Nanoemulsion. The selection of surfactant for the development of eucalyptus oil nanoemulsion was made based on the literature [22]. The selection of cosurfactant was made on the pseudoternary phase diagram, and finally, the ratio of surfactant and cosurfactant was done following evaluation of a wide range $(4: 1,3: 1,2: 1,1: 1,1: 2$, $1: 2,1: 3$, and $1: 4$ ) [23]. A preliminary study on the solubility of LUL was analysed in eucalyptus oil, surfactant (Tween 20), and PEG 200 (selected cosurfactant) to determine the maximum drug solubility within different components of nanoemulsion [24].

The formulation was developed following solubilization of the drug into the oil phase and mixed using a vortex mixer for $10 \mathrm{~min}$. The mixture of surfactant and cosurfactant (Smix) was then added to the drug solution in the oil followed by the addition of aqueous phase and high-speed homogenization $(6000 \mathrm{rpm})$ using Ultra Turrax ${ }^{\circledR}$ equipment (IKA, Germany) for 15 min under an ice bath to control the temperature. Finally, a clear transparent homogenous nanoemulsion was developed. 
2.3. Optimization of Nanoemulsion by Box-Behnken Statistical Design. Optimization of \% of oil, Smix, and stirring time for the nanoemulsion development was achieved using Box-Behnken statistical design (Design Expert ${ }^{\circledR}$, version 12; State-Ease Inc., USA). In the optimization process, three independent variables (percentage of oil, Smix, and homogenization time) were considered as three factors and the effect of their interaction at their three levels $(-1,0$, and 1$)$ on globule size and entrapment efficiency of the formulation was studied. The software suggested 17 batches of formulations with varying levels of three independent variables. The formulations were developed and the globule size and entrapment efficiency for all the 17 batches were determined following the methodology mentioned in Sections 2.4 and 2.5. The data were incorporated in the response column in the software to obtain the optimized formation. Statistical analysis was performed using analysis of variance (ANOVA) and the effect of the interaction of three independent variables at their different levels on the globule size and entrapment efficiency was analysed from the generated perturbation plots, contour plots, experimental versus predicted plots, and 3D surface plots [25, 26]. Quadratic equation generated by the best-fit quadratic model is depicted in

$$
\begin{aligned}
Y= & b 0+b 1 A+b 2 B+b 3 C+b 12 A B+b 13 A C \\
& +b 23 B C+b 11 A 2+b 22 B 2+b 33 C 2,
\end{aligned}
$$

where $Y$ represents measured responses, globule size, and entrapment efficiency whereas $b 0$ is the intercept and $b 1, b 2$, $b 3, b 12, b 13, b 23, b 11, b 22$, and $b 33$ are the regression coefficient for the model term of $A, B$, and $C$ and combination of them [27].
2.4. Determination of Dispersed Globule Size, Polydispersity Index, Surface Charge, and Morphology. The size of the dispersed globules and the PDI in the nanoemulsions was measured following dilution (50 times) in distilled water using the Zetasizer analyzer (Nano ZSP, Malvern, Worcestershire, UK) by measuring the changes in intensity of scattered light through the sample. The analysis was performed in triplicate at room temperature. The potential at the outside of the stationary layer of the dispersed globules was measured using the same Zetasizer instrument.

The morphology of the optimized nanoemulsion was determined using by polarized microscope (Nikon Instruments Inc. Melville, NY, US) and transmission electron microscopy (TEM) (JEOL JEM 1010, Tokyo, Japan). For TEM analysis, the drops of diluted samples were placed on a carbon grid and stained after drying the sample and analysed under electron microscopy operated at $80 \mathrm{KV}$ at $10000 \mathrm{X}$ magnification.

\subsection{Determination of Entrapment Efficiency of LUL within the} Formulated Nanoemulsion. The entrapment of LUL within the nanoemulsion formulation was determined following the method of Hussain and team [28]. This was done by removing the unentrapped drug from the developed nanoemulsion using the dialysis bag of 10,000 to $14,000 \mathrm{~mol}$ wt. cutoffs (Sigma Aldrich, MO, USA). The concentration of the drug was determined by the use of a UV-visible spectrophotometer at $\lambda \max 299 \mathrm{~nm}$ [6]. The following equation (equation (2)) was used to determine the entrapped quantity of LUL within the formulated nanoemulsion:

$$
\text { Entrapment efficiency }(\%)=\frac{\text { amount of drug determined in the formulation }}{\text { total drug added in the formulation }} \times 100 \text {. }
$$

2.6. Preparation Method of LUL-Loaded NEG. The optimized nanoemulsion containing the drug was developed using eucalyptus oil (15\%) and Smix $(3: 1)(45 \%)$ at a homogenization time of $15 \mathrm{~min}$. The NEG of the optimized nanoemulsion was developed by incorporating into Carbopol 934 to the nanoemulsion to get final polymer concentration of $0.5 \%(\mathrm{w} / \mathrm{w})$ [28]. Consistent dispersion of the polymer was prepared initially in distilled water to get the hydrogel of Carbopol 934. The dispersion was kept overnight at constant stirring using magnetic stirrer $(50 \mathrm{rpm})$ following the addition of triethanolamine (2-3 drops) to facilitate the formation of crosslinking between the polymeric components. Finally, the developed formulation contains an LUL concentration of $10 \mathrm{mg} / \mathrm{mL}$ in the fabricated LUL-NEG.

2.7. Determination of Viscosity, $p H$, and Refractive Index of the LUL Nanoemulgel. The $\mathrm{pH}$ of the fabricated LUL-loaded nanoemulgel was determined using calibrated $\mathrm{pH}$ meter, whereas the viscosity of the formulation was determined using a rotational viscometer at room temperature $\left(25 \pm 2^{\circ} \mathrm{C}\right)$. The refractive index of the optimized nanoemulgel was determined using an Abbe refractometer.

2.8. Determination of Rheological Property of the LUL Nanoemulgel. The rotational viscometer attached to the cone and plate of the Brookfield viscometer (Model DV-E, Brookfield, Middleboro, MA, USA) was used in this present study to determine the rheological properties of the LULNEG. The rheogram for the determination of viscosity was determined with the increasing shear rate from 0 to $200 \mathrm{~S}^{-1}$. The measured values of viscosity were (Pa.s) recorded at room temperature $\left(25 \pm 1^{\circ} \mathrm{C}\right)$ and plotted the viscosity versus shear rate curve to interpret our findings.

2.9. Determination of In Vitro Antifungal Activity Using the Well-Diffusion Method. Determination of antifungal efficacy of the LUL-NEG was compared with DMSO (5\%), 
blank NEG, LUL gel, and LUL solution following the method described by Kadimi and group [29]. In this well, diffusion method was used where the efficacy of the formulations was tested against Candida albicans. Initially, the organism was cultured in sabouraud dextrose agar media ( $\mathrm{pH}$ 6.2). Then, the media was prepared and sterilized using an autoclave $\left(121^{\circ} \mathrm{C}\right.$ for $\left.20 \mathrm{~min}\right)$. The media was then transferred into the sterile Petri dishes aseptically in laminar flow cabinet. Just before the addition of media, the grown culture was mixed with the normal media. The Petri dishes were incubated in inverted positions at $35^{\circ} \mathrm{C}$ for $48 \mathrm{~h}$ after an hour of loading the respective formulation into the wells prepared using a cork borer. The readings were recorded as the zone of inhibition by measuring the diameters.

\subsection{Animal-Based Study}

2.10.1. Animal Procurement. Procured experimental Wistar rats (180 to $220 \mathrm{~g}$ ) from registered breeder were acclimatized to the standard laboratory conditions $\left(25 \pm 2^{\circ} \mathrm{C}\right.$ and $55 \pm 5 \%$ relative humidity) for 7 days with free access to food and water. The animals were kept in polypropylene cages, which were maintained $12 \mathrm{~h}$ dark and light cycle under the standard condition. The experimental protocols were approved by the Research Ethics committee, approval number (PH-130-41), Faculty of Pharmacy, King Abdulaziz University, Jeddah, Saudi Arabia.

2.10.2. Ex Vivo Permeability Study. Ex vivo skin permeation study of the LUL-NEG and LUL gel formulations through the rat skin was studied using Franz diffusion cells. A freshly excised abdominal rat skin was collected after $24 \mathrm{~h}$ of removal of skin from the experimental animal. The freshly excised skin was washed with normal saline and the subcutaneous fat layer beneath the skin was removed. Thereafter, the skin was arranged between the donor and acceptor compartment, facing the stratum corneum of the skin towards the donor compartment and the dermis part touching the receptor compartment [28]. A $1 \mathrm{~mL}$ volume of the nanoemulgel was loaded to the $3.104 \mathrm{~cm}^{2}$ diffusion area and the setup was maintained at $34 \pm 1^{\circ} \mathrm{C}$ to mimic the skin condition. A magnetic bead was used to maintain constant stirring of the phosphate buffer $(\mathrm{pH}$ $7.4)$ in the receptor chamber. The samples $(0.5 \mathrm{~mL})$ were withdrawn from the receptor chamber of the diffusion cell at predetermined time intervals with the replacement of a similar volume of fresh buffer. The samples were analysed for LUL after filtration using HPLC with the mobile phase consisting of ammonium phosphate buffer $(0.1 \mathrm{M})$ and acetonitrile at $60: 40$ ratio [30]. Later, the permeation parameters (apparent coefficient of permeation (Papp) and flux (Jss)) were calculated using the following equations:

$$
\begin{aligned}
P_{\text {app }}= & \frac{\text { slope }}{\text { tissue surface area }} \\
& \times \text { volume of the donor compartment, }
\end{aligned}
$$

$$
\begin{aligned}
\text { Jss }= & P_{\text {app }} \\
& \times \text { concentration of LUL in the donor compartment. }
\end{aligned}
$$

2.10.3. Skin Irritation Studies. The irritation study of the developed LUL-NEG was determined in experimental Wistar rats [31]. The acclimatized animals were used to remove the hair from the dorsal side $24 \mathrm{~h}$ before the study, keeping precaution of not to damage the superficial layer of the skin. The animals were then divided into four groups, where the first group was left untreated (group I; control group) and the other three groups were treated with formalin solution $(0.8 \%)$ (group II, positive control), blank NEG (group III), and LUL-NEG (group IV), respectively. The formulations were applied on the cleaned skin over an area of $1 \mathrm{~cm}^{2}$ and a similar volume of blank NEG was applied in the drug-free treatment group. The skins of the experimental animals were observed at 24 and $48 \mathrm{~h}$ for any dermal reactions, that is, erythema or oedema scores. The score for severe erythema or oedema is 3 , where the corresponding scores of moderate, slight, and no erythema or oedema are 2 , 1 , and 0 , respectively.

2.10.4. Histopathological Assessment of Treated Rat Skin. A portion of rat skin was exposed to different treatments, that is, control group, formalin-treated (positive control), and LUL-NEG, to determine possible topical toxicity. The animals were sacrificed using an overdose of ketamine/ xylazine and the skin samples were collected by excision. Sample preparation and sectioning were carried out using a microtome. Furthermore, the sectioned samples were stained using haematoxylin and eosin dye. The staining of the samples helped in visualization of the cross-sectioned samples under microscopy. Each slide was properly visualized and imaged using an optical Leica microscope under 400 magnification.

2.11. Statistical Analysis. All the readings were measured in triplicate and the results were presented as mean \pm standard deviation. The analysis of the data was performed using oneway ANOVA followed by Tukey multiple comparison test to 
compare the groups of experimental results where $P<0.05$ was considered as statistically significant findings among the groups.

\section{Results and Discussion}

\subsection{Preparation of LUL-Loaded Nanoemulsion.} Preliminary screening on solubility study of LUL in the eucalyptus oil, Tween 20, and PEG 200 revealed $74.6 \pm 2.2 \mathrm{mg} / \mathrm{mL}, 68.3 \pm 1.7 \mathrm{mg} / \mathrm{mL}$, and $52.2 \pm 1.4 \mathrm{mg} / \mathrm{mL}$, respectively. The solubility results suggest that the desired concentration of LUL can easily be incorporated within the nanoemulsion formulation for delivery of LUL in the treatment of fungal infection. Among the tested cosurfactants (ethanol, carbitol, transcutol, and PEG 200) for the emulsification of eucalyptus oil with the help of Tween 20, we found that PEG 200 was most suitable with the higher area of nanoemulsion within the pseudoternary diagram (data not shown). Further, regarding the determination of ratio for the Smix for further processing of nanoemulsion development, we observed that $3: 1$ provides the highest area of nanoemulsion in the pseudoternary phase diagram. Therefore, the $25 \%$ cosurfactant in the Smix helps in increasing the fluidity of the surfactant significantly, which resulted in the highest nanoemulsion area in the pseudoternary phase diagram [32]. Selecting the ratio of $\operatorname{Smix}(3: 1)$, the optimization of drug-loaded nanoemulsion was performed in the next stage.

\subsection{Optimization of LUL-Loaded Nanoemulsion Using Box-Behnken Statistical Design}

3.2.1. Optimization for Globule Size of the Nanoemulsion. Globule size is one important measure for the nanoemulsion formulation as it provides the stability, the aesthetic appeal of the nanoemulsion together with penetration through the skin $[13,33]$. Therefore, the software-based optimization process was adopted in this study to optimize the globule size to reduce the experimental burden. The compositions of seventeen formulations recommended by the software are presented in Table 1. The statistical results on the effect of the interaction of process parameters such as homogenization time and the important formulation components such as oil $\%$ and \% of Smix on globule size of LUL-loaded nanoemulsions are presented in Table 2. From the representation, it could be said that the model terms $\mathrm{A}, \mathrm{B}, \mathrm{C}, \mathrm{A}^{2}$, and $\mathrm{B}^{2}$ have a statistically significant influence $(P$ values $<0.05)$ on the globule size of the developed formulations.

The model $F$-value of 85.75 and $P$ value of $<0.05$ represented the significance of the used quadratic model. The predicted $R^{2}(0.8585)$ and adjusted $R^{2}(0.9795)$ values are in reasonable agreement with a difference of less than 0.2 . Additionally, the desirable value for adequate precision (signal-to-noise ratio) should be greater than 4 , whereas the adequate precision value of 28.962 indicated an adequate signal in the used model. Hence, this model could be used to navigate the design space. The observed and predicted values for the globule size are also in close agreement as depicted in Table 1.
A polynomial equation on the effect of the interaction of three independent variables on globule size of the developed nanoemulsion formulations was generated in the fitted model (equation (5)), where coefficient values for the model terms A $(+13.68)$ and $\mathrm{B}(+11.01)$ are positive, which indicated that the globule size will increase with increasing $\%$ of oil and Smix, respectively. On the contrary, the negative coefficient value of -8.06 for the model term $\mathrm{C}$ indicated that increasing homogenization time resulted in decreasing globule size of the nanoemulsion. The increasing globule size with increasing oil \% is in agreement with reported data $[34,35]$.

$$
\begin{aligned}
Y 1 & =+16.33+13.68 * A+11.01 * B-8.06 * C \\
& +20.60 * A^{2}-3.78 * A * B+1.73 * A * C \\
& +31.13 * B_{2}+2.79 * B * C-1.45 * C^{2} .
\end{aligned}
$$

Further, the initial decrease followed by an increase in globule size of the nanoemulsion with increasing \% of oil and Smix is evident in the perturbation plot and 3D surface plot (Figures $1(\mathrm{a})$ and $1(\mathrm{~b})$ ). The initial increase in oil \% may lead to proper solubilization of LUL, which might result in a decrease in globule size, and a further increase in oil \% resulted in an increase in globule size due to insufficient surfactant to effectively coat the dispersible globule. A decrease in globule size with an initial increase in surfactant \% was noted which might be due to a reduction in interfacial tension between the dispersible globules and aqueous phase and provide a successful coating over the dispersible globules [34], whereas a further increase in surfactant concentration leads to an increase in globule size which might be due to formation aggregation after a certain \% of Smix concentration. Increasing stirring time (model term $\mathrm{C}$ ) resulted in decreasing the globule size, which is reflected in equation (5) by the negative coefficient value for model term $\mathrm{C}$ and also in perturbation plot (Figure 1(a)) with decreasing slope associated with increasing stirring time. Our findings are in agreement with the existing reported data [36]. As mentioned previously, all three model terms have a significant effect on the globule size of the formulation (Table 2). Predicted and experimental data for the globule size are in close agreement, which can be seen in the predicted versus actual plot (Figure 1(c)) and also in Table 1.

\subsubsection{Optimization for Entrapment Efficiency of the} Nanoemulsion. Higher \% of encapsulation efficiency is one of the major targets in formulation development. During the optimization process of encapsulation efficiency of nanoemulsion formulation, the statistical outcome (Table 2) on the interaction of three independent variables indicated that model terms $A, C, A C, A^{2}, B^{2}$, and $C^{2}$ are significant $(P<0.05)$.

Further, the $F$-value of 122.76 of the model and the $P$ value of $<0.05$ indicated the significance of the used quadratic model. The predicted $R^{2}(0.9587)$ and adjusted $R^{2}$ $(0.9856)$ values are in close agreement as the difference is less than 0.2. Additionally, an adequate precision value of 32.838 
TABLE 1: Box-Behnken statistical design: levels of three independent variables from the experimental runs along with the predicted responses on LUL-loaded nanoemulsion.

\begin{tabular}{|c|c|c|c|c|c|c|c|}
\hline \multirow[b]{2}{*}{ Batch } & \multicolumn{3}{|c|}{ Levels of independent variables } & \multicolumn{2}{|c|}{ Actual responses } & \multicolumn{2}{|c|}{ Predicted responses } \\
\hline & $A(\% \mathrm{v} / \mathrm{v})$ & $B(\% \mathrm{v} / \mathrm{v})$ & $C(\% \mathrm{v} / \mathrm{v})$ & $\begin{array}{l}\text { Globule size } \\
(Y 1)(\mathrm{nm})\end{array}$ & $\begin{array}{c}\text { Entrapment efficiency } \\
Y 2(\%)\end{array}$ & $\begin{array}{l}\text { Globule size } \\
(Y 1)(\mathrm{nm})\end{array}$ & $\begin{array}{c}\text { Entrapment efficiency } \\
Y 2(\%)\end{array}$ \\
\hline $\mathrm{F} 1$ & 0 & -1 & 1 & 20.62 & 85.23 & 24.13 & 85.3 \\
\hline $\mathrm{F} 2$ & 0 & 0 & 0 & 16.67 & 92.86 & 16.33 & 92.74 \\
\hline F3 & 1 & 1 & 0 & 87.32 & 93.51 & 88.96 & 93.94 \\
\hline $\mathrm{F} 4$ & 0 & 1 & 1 & 54.02 & 86.54 & 46.87 & 86.19 \\
\hline F5 & 0 & 0 & 0 & 15.77 & 93.46 & 16.33 & 92.74 \\
\hline F6 & 1 & 0 & -1 & 53.61 & 95.34 & 55.48 & 94.98 \\
\hline F7 & 0 & 0 & 0 & 17.23 & 91.79 & 16.33 & 92.74 \\
\hline F8 & 0 & 0 & 0 & 15.89 & 92.44 & 16.33 & 92.74 \\
\hline F9 & -1 & 0 & -1 & 32.21 & 85.45 & 31.56 & 85.53 \\
\hline F10 & 1 & -1 & 0 & 78.63 & 93.56 & 74.49 & 93.57 \\
\hline F11 & 1 & 0 & 1 & 42.18 & 94.48 & 42.81 & 94.4 \\
\hline $\mathrm{F} 12$ & -1 & -1 & 0 & 41.23 & 82.46 & 39.59 & 82.03 \\
\hline F13 & -1 & 0 & 1 & 13.88 & 79.33 & 12.01 & 79.69 \\
\hline $\mathrm{F} 14$ & -1 & 1 & 0 & 65.02 & 81.32 & 69.16 & 81.31 \\
\hline F15 & 0 & -1 & -1 & 43.57 & 89.22 & 45.84 & 89.57 \\
\hline F16 & 0 & 1 & -1 & 65.79 & 88.39 & 62.28 & 88.32 \\
\hline F17 & 0 & 0 & 0 & 16.07 & 93.14 & 16.33 & 92.74 \\
\hline \multicolumn{4}{|c|}{ Independent variable } & & Low $(-1)$ & $\begin{array}{c}\text { Levels } \\
\text { Medium (0) }\end{array}$ & High (1) \\
\hline \multicolumn{4}{|c|}{$A=$ oil $(\% v / v)$} & & 10 & 15 & 20 \\
\hline \multicolumn{4}{|c|}{$B=\operatorname{Smix}(\% v / v)$} & & 35 & 45 & 55 \\
\hline \multicolumn{4}{|c|}{$C=$ homogenization time $(\mathrm{min})$} & & 10 & 15 & 20 \\
\hline $\begin{array}{l}\text { Depenc } \\
Y 1=\mathrm{gl} \\
Y 2=\mathrm{er}\end{array}$ & $\begin{array}{l}\text { nt variables } \\
\text { ule size (nm } \\
\text { apment effic }\end{array}$ & cy (\%) & & & & & \\
\hline
\end{tabular}

TABLE 2: Analysis of variance data for globule size and entrapment efficiency.

\begin{tabular}{lccccc}
\hline \multicolumn{3}{l}{ ANOVA on globule size } & \multicolumn{3}{c}{$\begin{array}{c}\text { ANOVA on entrapment } \\
\text { efficiency }\end{array}$} \\
Source & $F$-ratio & $P$ value & Source & $F$-ratio & $P$ value \\
\hline Model & 85.75 & $<0.0001$ & Model & 122.76 & $<0.0001$ \\
$A$ & 124.66 & $<0.0001$ & $A$ & 792.44 & $<0.0001$ \\
$B$ & 80.84 & $<0.0001$ & $B$ & 0.1710 & 0.6916 \\
$C$ & 43.30 & 0.0003 & $C$ & 55.76 & 0.0001 \\
$A B$ & 4.75 & 0.0657 & $A B$ & 0.8061 & 0.3991 \\
$A C$ & 0.9918 & 0.3525 & $A C$ & 18.77 & 0.0034 \\
$B C$ & 2.60 & 0.1506 & $B C$ & 3.11 & 0.1213 \\
$A^{2}$ & 148.84 & $<0.0001$ & $A^{2}$ & 39.55 & 0.0004 \\
$B^{2}$ & 339.93 & $<0.0001$ & $B^{2}$ & 114.49 & $<0.0001$ \\
$C^{2}$ & 0.7407 & 0.4179 & $C^{2}$ & 56.71 & 0.0001 \\
\hline
\end{tabular}

indicated an adequate signal for the used model. Hence, this model could be used to navigate the design space.

A polynomial equation on the effect of the interaction of three independent variables on entrapment efficiency of the developed nanoemulsion formulations was generated (equation (6)). The positive coefficient value for the model terms $A(+6.04)$ indicated that the entrapment efficiency would increase with an increasing $\%$ of oil. On the contrary, the negative coefficient of model terms $B(-0.0887)$ and $C$ $(-1.60)$ indicated that the increasing \% of Smix and stirring time would lead to decreasing in entrapment efficiency of the LUL nanoemulsion. However, Smix does not have a significant effect on entrapment efficiency as indicated by $P$ values in Table 2.

$$
\begin{aligned}
Y 1= & +92.74+6.04 * A-0.0887 * B-1.60 * C-1.86 * A^{2} \\
& +0.2725 * A * B+1.32 * A * C-3.17 * B^{2} \\
& +0.5350 * B * C-2.23 * C^{2} .
\end{aligned}
$$

Increasing the entrapment efficiency with increasing oil $\%$ is further confirmed in perturbation plot (Figure 2(a)) with positive slope associated with increasing oil \%, in contour plot (Figure 2(b)) with colour changes from blue to red with increasing oil \%. A similar effect of oil on entrapment efficiency is evident in the 3D surface plot (Figure 2(c)). A higher \% oil supports solubilization of the entrapped drug, which might help in enhancing the entrapment efficiency of the LUL in the formulation, whereas increasing stirring time resulted in decreasing entrapment efficiency, which could be represented by the negative slope associated with model term C (Figure 2(a)), and the finding 


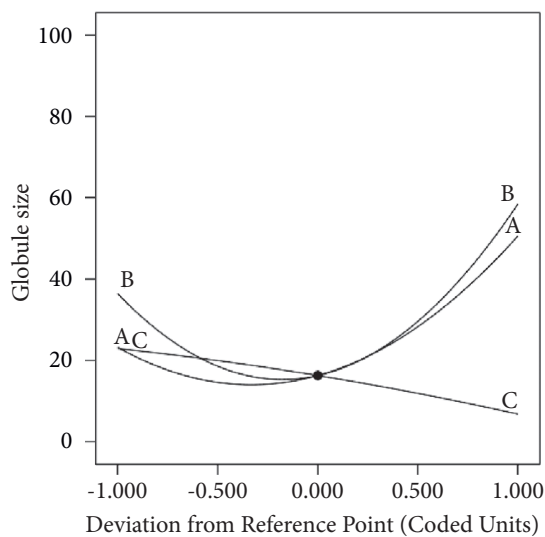

(a)

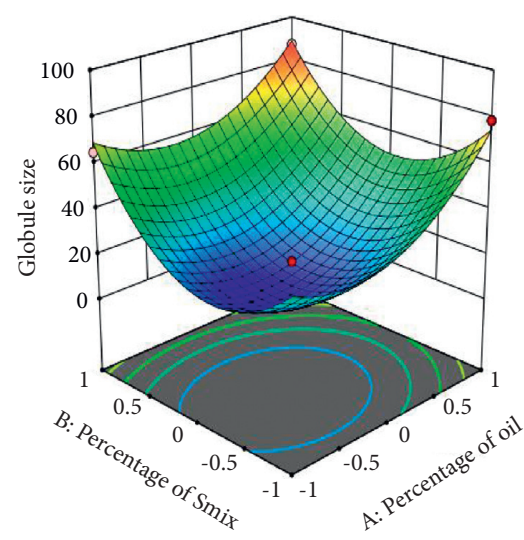

(b)

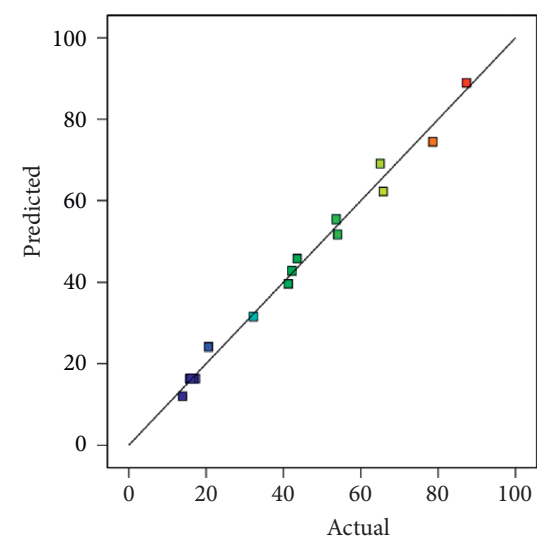

(c)

FIGURE 1: Consequence of independent variables on globule size. Results were presented in the (a) perturbation plot, (b) 3D surface plot, and (c) predicted versus actual graph.

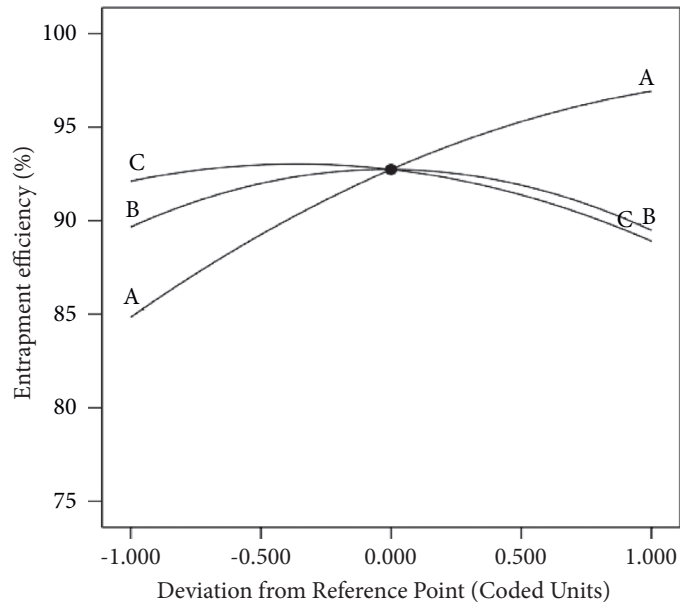

(a)

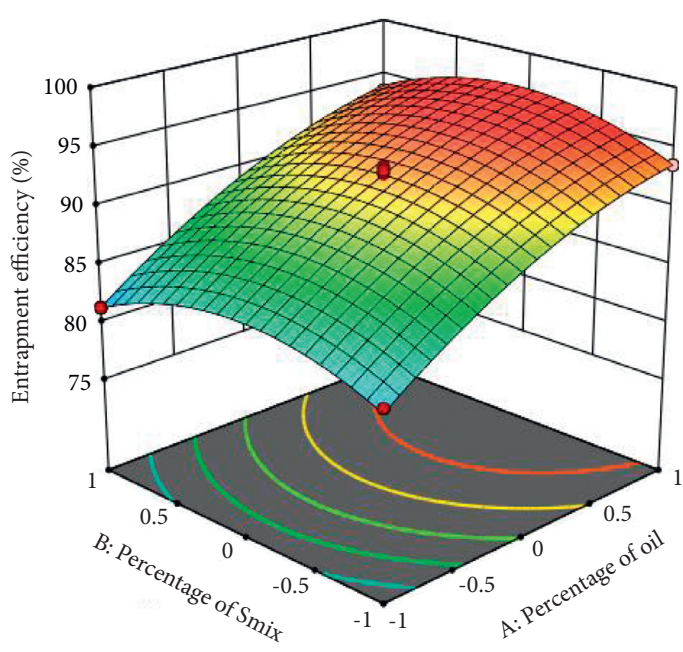

(c)

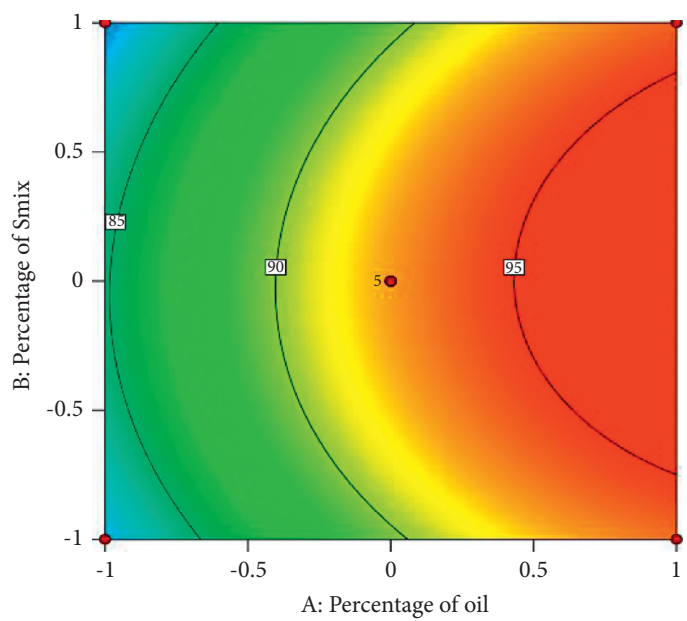

(b)

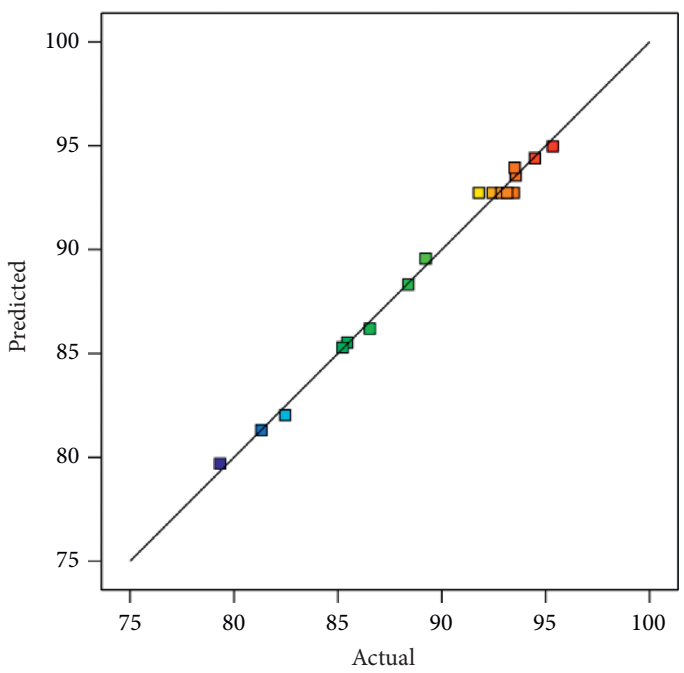

(d)

Figure 2: Consequence of independent variables on entrapment efficiency. Results were presented in the (a) perturbation plot, (b) contour plot, (c) 3D surface plot, and (d) predicted versus actual graph. 
is at per the negative coefficient of stirring time in equation (6). A similar effect of stirring time on entrapment efficiency is reported in the literature [36]. The effect of \% oil and stirring time on entrapment efficiency was further confirmed in the 3D surface plot (Figure 2(c)). The closeness of experimental and predicted values for the entrapment efficiency is represented in Table 1 and the predicted versus actual plot (Figure 2(d)).

3.3. Characterization of the Formulation for Globule Size, PDI, Morphology, and Zeta Potential. Being an important characterizing parameter of nanoemulsion, the size of the dispersed globules provides the stability of the formulation, whereas the nanometric lipid globule allows easy penetration from the stratum corneum. Alternatively, the PDI of the dispersion refers to the uniformity of the dispersed globule size, where the PDI $<0.5$ is considered as homogenous distribution $[13,32]$. The results of the optimized nanoemulsion were found to be $16.67 \pm 3.67 \mathrm{~nm}$, where the PDI was $0.376 \pm 0.022$, indicating the usefulness of our drugloaded nanoemulsion for topical application [37].

The zeta potential provides the charge on the globule surface that contributes to the stability of the formulation, where higher charges allow repulsive forces between the globules and thus prevent aggregation or coalition of the dispersed globules [38]. However, the zeta potential of the dispersed globules was found to be $-9.53 \pm 0.25 \mathrm{mV}$. This low zeta potential is due to the nonionic surfactant incorporated in our formulation. Incorporation of these globules in the polymeric matrix will hinder the movement of the globules, thereby imparting the stability of the formulation [15]. Further, the negative charge of the dispersed drugloaded oil globules might be due to the presence of anionic groups in the cosurfactant and oil core [14].

Further, analysis of morphology and globule size of the developed formulation as obtained under a polarized microscope and TEM study are presented in Figures 3(a) and 3(b). The polarized microscopic and TEM micrographs represented the spherical morphology of the dispersed globules in the system. Further, the size of the globules obtained from the dynamic light scattering method is in agreement with the size found in the TEM micrograph. Our results on the morphology of the formulation are as per our previous findings [14].

3.4. Characterization of the LUL-NEG for Viscosity, $p H$, and Refractive Index. Carbopol is a water-soluble cross-linked polyacrylic acid polymer, widely used in topical preparation as a gelling agent. This $\mathrm{pH}$-sensitive polymer has also gained popularity in pharmaceutical products as suspending, stabilizing, and emulsifying agent. The addition of triethanolamine helps to neutralize the developed formulation to form into gel at skin $\mathrm{pH}$ [39]. The incorporation of the formulated and optimized nanoemulsion in the prepared gel matrix of $0.5 \%(\mathrm{w} / \mathrm{w})$ Carbopol 934 at a ratio of $1: 1$ produced the final NEG containing LUL.

The $\mathrm{pH}$ of the NEG to be applied on skin should be compatible; thus, determination of $\mathrm{pH}$ is important. The determination of $\mathrm{pH}$ of the developed LUL-NEG was found to be $5.65 \pm 0.17$, almost comparable to the $\mathrm{pH}$ of the skin. Thus, it could be said that the $\mathrm{pH}$ of the formulation would be favorable to the patients [14]. Further, the viscosity of the LUL-NEG was found to be $9.26 \pm 0.08 \mathrm{~Pa}$.s at a shear stress of $60 \mathrm{~Pa}$ and shear rate of $6 \mathrm{~s}^{-1}$. It was found that the incorporation of optimized nanoemulsion into the blank NEG did not alter the rheological property of the formulation. Our results are in agreement with our previous findings [14], which suggest that the viscosity of the product would be in a stage to easily spread over the skin.

Further, the refractive index is an optical property where the isotropic nature of the formulation reflects no interaction between the drug and excipient. The refractive index of our optimized LUL-NEG formulation was found to be $1.31 \pm 0.08$, which is quite similar to water representing clear and transparent hydrogel without any interaction.

3.5. Rheological Property of the LUL-NEG. Two important parameters, adherence and spreading, are essential for the formulations to be applied topically. After equilibration of the formulation for a week, the curve was plotted to represent the relationship between shear rate and the applied stress (Figure 4). The experimental results of viscosity (Pa.s) of the LUL-NEG were found to be thixotropic characteristics with the increase in shear within the range of 20 to $200 \mathrm{~S}^{-1}$. The descending lines of the presented curves (Figure 4) confirmed it. The rheological properties of the gel formulations are correlated to the preparation process, the molecular weight of the incorporated polymer, and the degree of crosslinking [40]. Based on the findings of the present investigation, it could be said that the rheological property of the formulation could be easily applied topically as the increasing rate of shear to the formulation resulted in a decrease in viscosity [41]. Therefore, to initiate a flow of the formulation, stress needs to be applied, which will facilitate easy spreadability over the topical area.

3.6. In Vitro Antifungal Efficacy. The results of the in vitro antifungal efficacy of DMSO (5\%), blank NEG, LUL solution, LUL gel, and LUL-NEG using well-diffusion technique are presented in Figures 5(a) and 5(b). The developed and commercial NEG formulations of LUL were found to be sensitive against strain of tested fungi within the limit of our experiment. The LUL-NEG was found to inhibit the growth of $C$. albicans significantly $(P<0.05)$ when compared with other four groups (Figure 5(b)). Significant efficacy of the developed LUL-NEG formulation over LUL solution and LUL gel might be explained by the fact of incorporating eucalyptus oil in the LUL-NEG, which potentiated the efficacy of the antifungal agent. This might be due to the inherent antifungal effect of eucalyptus oil [20,21], which was reflected by the zone of inhibition by blank nanoemulsion. Thus, the increased inhibitory effect of LUL-NEG is due to the synergistic role of LUL and eucalyptus oil when codelivered through nanoemulsion platform, which allowed intense diffusion of the drug containing oil globules. A comparable result is available in the literature where the 


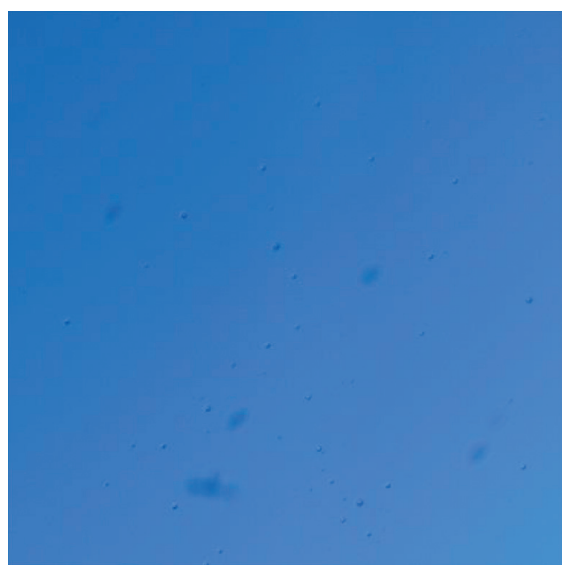

(a)

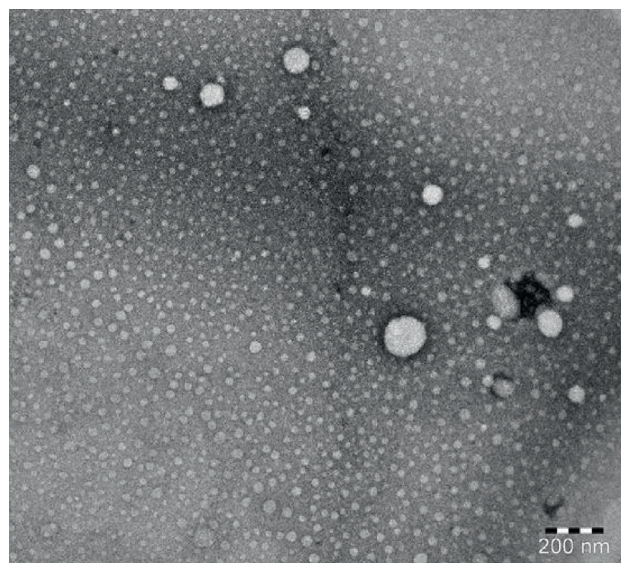

(b)

Figure 3: Presentation on morphology of the optimized formulation under the polarized microscope (a) and transmission electron microscope (b).

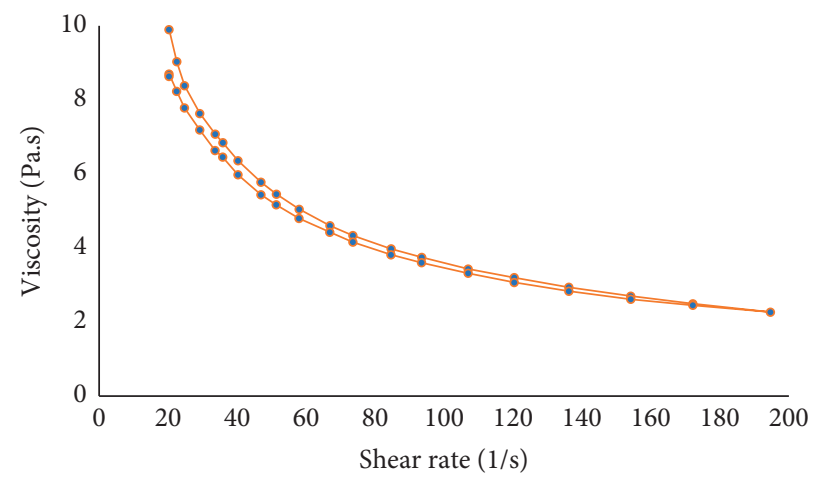

FIGURE 4: Viscosity versus shear rate profile of the fabricated LUL-NEG.

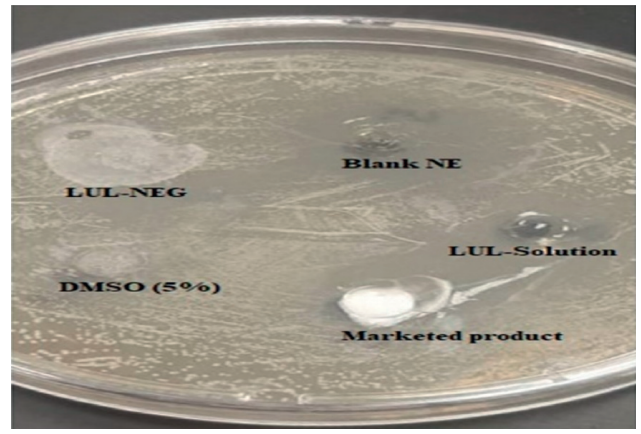

(a)

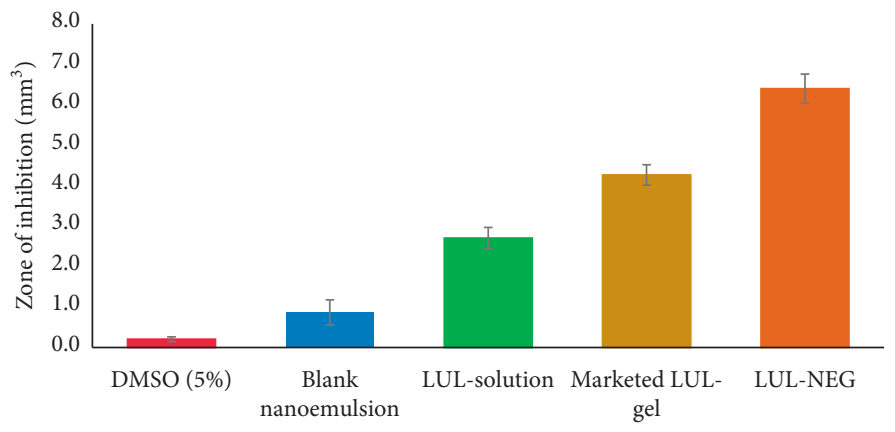

(b)

Figure 5: Areas of zone of inhibition in the tested samples in the Petri dish containing Candida albicans (a) and the presentation of the measured area of the zone of inhibition of different groups (b). The results were presented as mean $\pm \mathrm{SD}(n=3) .{ }^{*} P<0.05$.

authors compared the microemulsion of LUL with the commercial formulation and reported statistically significant inhibition of growth with LUL microemulsion when compared to commercial formulation [42]. Therefore, the nanoemulsion platform containing eucalyptus oil as the oil core for LUL was found to possess superior efficacy against C. albicans species.
3.7. Ex Vivo Skin Permeation Study. The results of ex vivo permeation of LUL from the LUL-NEG and LUL gel preparation are presented in Figure 6. From the comparison on permeation profile of LUL from the two formulations, it is clearly observed that the permeation of LUL from the developed NEG formulation $\left(406.2 \pm 18.6 \mu \mathrm{g} / \mathrm{cm}^{2}\right)$ is significantly higher $(P<0.05)$ when compared to the LUL gel 


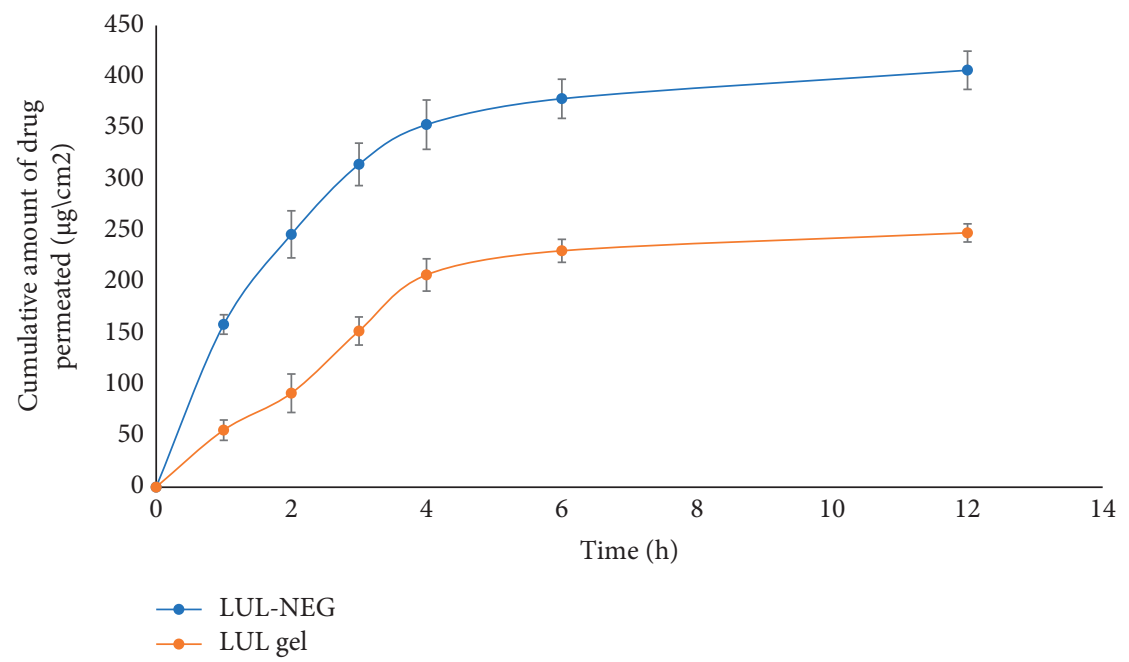

Figure 6: Cumulative amount of LUL permeation from LUL-NEG and LUL gel across the experimental rat skin. The results were presented as mean $\pm \mathrm{SD}(n=3)$.

formulation $\left(247.7 \pm 8.8 \mu \mathrm{g} / \mathrm{cm}^{2}\right)$ (Figure 6). This increased permeation might be due to the presence of nanosized oil globules containing LUL, which could increase the rate of permeation from the lipophilic layers of the skin to facilitate the permeation of the drug across the skin without any lag time $[43,44]$. Simultaneously, when the permeation parameters were compared, it was found that the permeation flux was significantly enhanced $(P<0.05)$ with LUL-NEG $\quad\left(37.098 \pm 1.05 \mu \mathrm{g} \mathrm{cm}^{-2} \mathrm{~h}^{-1}\right)$ when compared to the gel formulation $\left(25.37 \pm 1.21 \mu \mathrm{g} \mathrm{cm}^{-2} \mathrm{~h}^{-1}\right)$. A similar result was observed when the permeation coefficient of LUL-NEG $\left(1.289 \times 10^{-4} \mathrm{~cm}^{-2} \mathrm{~h}^{-1}\right)$ and LUL gel $\left(0.880 \times 10^{-4} \mathrm{~cm}^{-2} \mathrm{~h}^{-1}\right)$ was compared. Finally, the apparent permeation and flux of the two formulations were compared and it was found that there is an enhancement ratio of 1.46 when compared the LUL-NEG to the LUL gel formulation. This might be due to the increase of skin permeation attributable to the presence of PEG 200 [45], whereas the nanometric lipophilic globules also facilitated such permeation.

3.8. Skin Irritation Study. Compliance of the patient is of utmost importance when a novel approach of formulation is made. Any signs of irritation might restrict the use of the formulation in patients; thus, the topical preparations should be free from any irritation. Thus, to evaluate the skin irritation, the fabricated LUL-NEG was tested and compared with other groups of treated animals. The outcome of the irritation study in different groups of animals is presented in Table 3. The results of the formulations containing LUL, the fabricated and commercial, did not show any signs of irritation (erythema (redness) and edema (swelling)) even after $48 \mathrm{~h}$ of exposure. Alternatively, the animals in the positive control group (Group II) showed signs of irritations with a score of 2 and 3 after $24 \mathrm{~h}$ and $48 \mathrm{~h}$ of exposure, respectively [28]. Thus, it indicated that the application of formalin to the skin of the experimental animals resulted in redness and itching reaction. On the other way, the animals
TABLE 3: Erythema/oedema scores of the skin in treated animals after 24 and $48 \mathrm{~h}$.

\begin{tabular}{lccc}
\hline Groups & Treatment & \multicolumn{2}{c}{ Erythema } \\
& & $24 \mathrm{~h}$ & $48 \mathrm{~h}$ \\
\hline Control group & No treatment & 0 & 0 \\
Group II & $(0.8 \%$ formalin solution $)$ & 2 & 3 \\
Group III & Blank NEG & 0 & 0 \\
Group IV & LUL-NEG & 0 & 0 \\
\hline
\end{tabular}

in groups I and III did not show any signs of erythema. Thus, from this study, it could be concluded that the polymeric blank NEG and LUL-NEG did not exhibit any signs of inflammation or irritation possessing safe delivery of the formulation to the skin for the treatment of any fungal infection.

3.9. Histopathological Study. To rule out any possible toxicity of the optimized formulation LUL-NEG, histopathological assessment was performed. The rat skin was treated with formalin solution as a positive control; blank NEG and LUL-NEG were compared with the control group. The examination of H\&E-stained sections of control rats' skin which is formed of 3-4 layers of keratinized stratified squamous cells with normally appearing keratin layer. Furthermore, the four distinct layers of epidermis consisted of keratinized stratified squamous epithelium cells, where the layers are separated by the name, stratum corneum, stratum granulosum, stratum spinosum, and stratum basale, from outside to inside. The junction of the epidermal-dermal depicted several epidermal elevations and dermal papillae. Alternatively, the other layer, dermis, consisted of 2 layers, papillary (thin, present directly below the epidermis) and reticular layer (thick, dense irregular connective tissue). The sweat gland and sebaceous, hair follicle were seen in the dermis (Figure $7(\mathrm{a})$ ). The formalintreated group showed pathological changes such as the 


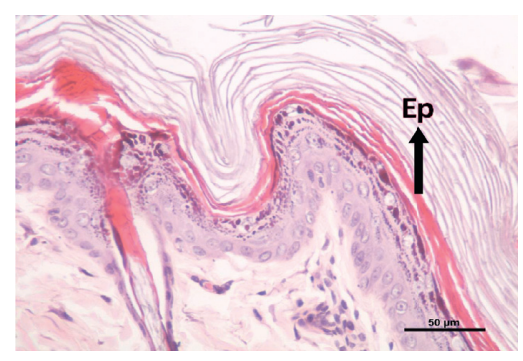

(a)

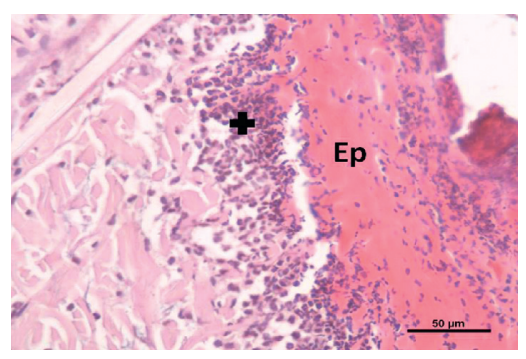

(b)

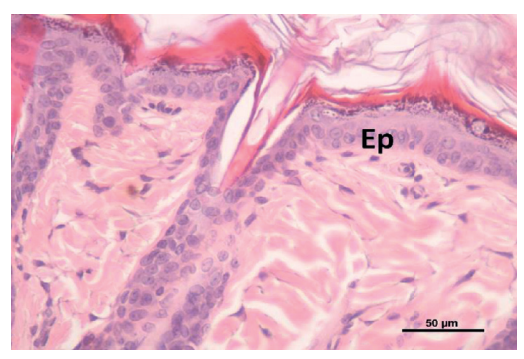

(c)

Figure 7: H\&E staining of control skin of the rat (a) showed 3-4 layers of keratinized stratified squamous cells with normally appearing keratin layer epidermis (Ep). Formalin exposed rat skin (b) showed thickened degenerated epidermis (Ep) and inflammatory cells infiltrate $(+)$, and LUL-NEG (c) exposed rat skin revealed no abnormal changes in the treated rat skin tissue as compared to controls (400x).

thickened degenerated epidermis (Ep), intercellular edema, and inflammatory cells infiltrate (Figure 7(b)). The LULNEG-treated groups (Figure $7(\mathrm{c})$ ) revealed no abnormal changes in the treated rat skin tissue as compared to controls, except moderately thickened epidermis. The overall results indicated that the LUL-NEG was within the limit of the skin tolerance and safe to use for topical applications.

\section{Conclusion}

LUL, a broad-spectrum antifungal agent, possesses permeability and retention issues with commercially available products. Thus, the present approach of improving penetration is using the nanoemulsion platform where the mucoadhesive polymer helped to develop the NEG formulation. The development of nanoemulsion was optimized using Box-Behnken statistical design. The ex vivo skin permeability of LUL from the developed NEG was found to be higher when compared to the commercial formulation; thus, there was $46 \%$ improvement in flux and Papp with LUL-NEG when compared with the commercial product. Incorporation of eucalyptus oil in the preparation revealed synergistic inhibition of the tested fungi, where improved diffusion of the oil globules from the NEG facilitated the efficacy. The prepared formulation was found to be safe when applied topically on the experimental animal. There were no signs of erythema in the LUL-NEG-administered animals. Furthermore, the histopathological study demonstrated a lack of toxicity on the skin of the experimental animals, suggesting the safe and efficacious cutaneous application of the formulation. Hence, from the obtained results, it could be concluded that the LUL-NEG would be an effective approach for localized delivery of LUL safely with improved efficacy.

\section{Data Availability}

The data used to support the findings of this study are included within the article.

\section{Conflicts of Interest}

The authors declare that they have no conflicts of interest.

\section{Acknowledgments}

This study was funded by the Deanship of Scientific Research (DSR) at King Abdulaziz University, Jeddah, under grant no. RG-11-166-38. The authors, therefore, acknowledge DSR with thanks for technical and financial support.

\section{References}

[1] D. Khanna and S. Bharti, "Luliconazole for the treatment of fungal infections: an evidence-based review," Core Evidence, vol. 9, pp. 113-124, Sep. 2014.

[2] S. Baghel, V. S. Nair, A. Pirani et al., "Luliconazole-loaded nanostructured lipid carriers for topical treatment of superficial Tinea infections," Dermatologic Therapy, vol. 33, no. 6, Article ID e13959, 2020.

[3] M. H. Gold and J. T. Olin, "Once-daily luliconazole cream 1\% for the treatment of interdigital tinea pedis," Expert Review of Anti-infective Therapy, vol. 13, no. 12, pp. 1433-1440, 2015.

[4] H. Koga, Y. Nanjoh, K. Makimura, and R. Tsuboi, "In vitroantifungal activities of luliconazole, a new topical imidazole," Medical Mycology, vol. 47, no. 6, pp. 640-647, 2009.

[5] H. Jerajani, C. Janaki, S. Kumar, and M. Phiske, "Comparative assessment of the efficacy and safety of sertaconazole (2\%) cream versus terbinafine cream (1\%) versus luliconazole (1\%) cream in patients with dermatophytoses: a pilot study," Indian Journal of Dermatology, vol. 58, no. 1, pp. 34-38, 2013.

[6] M. Kumar, N. Shanthi, A. K. Mahato, S. Soni, and P. S. Rajnikanth, "Preparation of luliconazole nanocrystals loaded hydrogel for improvement of dissolution and antifungal activity," Heliyon, vol. 5, no. 5, Article ID e01688, 2019.

[7] M. Kaur, K. Singh, and S. K. Jain, "Luliconazole vesicular based gel formulations for its enhanced topical delivery," Journal of Liposome Research, vol. 30, no. 4, pp. 388-406, 2020.

[8] A. Mahmood, V. K. Rapalli, T. Waghule, S. Gorantla, and G. Singhvi, "Luliconazole loaded lyotropic liquid crystalline nanoparticles for topical delivery: QbD driven optimization, in-vitro characterization and dermatokinetic assessment," Chemistry and Physics of Lipids, vol. 234, Article ID 105028, 2021.

[9] A. K. Garg, B. Maddiboyina, M. H. S. Alqarni et al., "Solubility enhancement, formulation development and antifungal activity of luliconazole niosomal gel-based system," Journal of Biomaterials Science, Polymer Edition, vol. 32, no. 8, pp. 1009-1023, 2021.

[10] S. Firdaus, N. Hassan, M. A. Mirza et al., "FbD directed fabrication and investigation of luliconazole based SLN gel for 
the amelioration of candidal vulvovaginitis: a $2 \mathrm{~T}$ (thermosensitive \& transvaginal) approach," Saudi Journal of Biological Sciences, vol. 28, no. 1, pp. 317-326, 2021.

[11] H. Choudhury, B. Gorain, M. Pandey et al., "Recent update on nanoemulgel as topical drug delivery system," Journal of Pharmaceutical Sciences, vol. 106, no. 7, pp. 1736-1751, 2017.

[12] S. H. Akrawi, B. Gorain, A. B. Nair et al., "Development and optimization of naringenin-loaded chitosan-coated nanoemulsion for topical therapy in wound healing," Pharmaceutics, vol. 12, no. 9, p. 893, 2020.

[13] L. Y. Chin, J. Y. P. Tan, H. Choudhury, M. Pandey, S. P. Sisinthy, and B. Gorain, "Development and optimization of chitosan coated nanoemulgel of telmisartan for intranasal delivery: a comparative study," Journal of Drug Delivery Science and Technology, vol. 62, Article ID 102341, 2021.

[14] S. Md, N. A. Alhakamy, H. M. Aldawsari et al., "Improved analgesic and anti-inflammatory effect of diclofenac sodium by topical nanoemulgel: formulation development-in vitro and in vivo studies," Journal of Chemistry, vol. 2020, Article ID 4071818, 10 pages, 2020.

[15] E. Yeo, C. J. Yew Chieng, H. Choudhury, M. Pandey, and B. Gorain, "Tocotrienols-rich naringenin nanoemulgel for the management of diabetic wound: fabrication, characterization and comparative in vitro evaluations," Current Research in Pharmacology and Drug Discovery, vol. 2, Article ID 100019, 2021.

[16] R. L. Rajput, J. S. Narkhede, A. Mujumdar, and J. B. Naik, "Synthesis and evaluation of luliconazole loaded biodegradable nanogels prepared by $\mathrm{pH}$-responsive Poly (acrylic acid) grafted Sodium Carboxymethyl Cellulose using amine based cross linker for topical targeting: in vitro and Ex vivo assessment," Polymer-Plastics Technology and Materials, vol. 59, no. 15, pp. 1654-1666, 2020.

[17] J. Wang, X. Wang, G. Yan, S. Fu, and R. Tang, "pH-sensitive nanogels with ortho ester linkages prepared via thiol-ene click chemistry for efficient intracellular drug release," Journal of Colloid and Interface Science, vol. 508, pp. 282-290, 2017.

[18] P. Sinha, S. Srivastava, N. Mishra et al., "Development, optimization, and characterization of a novel tea tree oil nanogel using response surface methodology," Drug Development and Industrial Pharmacy, vol. 42, no. 9, pp. 1434-1445, 2016.

[19] M. E. Elmataeeshy, M. S. Sokar, M. Bahey-El-Din, and D. S. Shaker, "Enhanced transdermal permeability of Terbinafine through novel nanoemulgel formulation; development, in vitro and in vivo characterization," Future Journal of Pharmaceutical Sciences, vol. 4, no. 1, pp. 18-28, 2018.

[20] H. Ramezani, H. P. Singh, D. R. Batish, and R. K. Kohli, "Antifungal activity of the volatile oil of Eucalyptus citriodora," Fitoterapia, vol. 73, no. 3, pp. 261-262, 2002.

[21] S. d. S. Gündel, S. N. de Godoi, R. C. V. Santos et al., "In vivo antifungal activity of nanoemulsions containing eucalyptus or lemongrass essential oils in murine model of vulvovaginal candidiasis," Journal of Drug Delivery Science and Technology, vol. 57, Article ID 101762, 2020.

[22] T. Adak, N. Barik, N. B. Patil et al., "Nanoemulsion of eucalyptus oil: an alternative to synthetic pesticides against two major storage insects (Sitophilus oryzae (L.) and Tribolium castaneum (Herbst)) of rice," Industrial Crops and Products, vol. 143, Article ID 111849, 2020.

[23] H. Choudhury, B. Gorain, S. Karmakar et al., "Improvement of cellular uptake, in vitro antitumor activity and sustained release profile with increased bioavailability from a nanoemulsion platform," International Journal of Pharmaceutics, vol. 460, no. 1-2, pp. 131-143, 2014.
[24] B. Gorain, H. Choudhury, E. Biswas, A. Barik, P. Jaisankar, and T. K. Pal, "A novel approach for nanoemulsion components screening and nanoemulsion assay of olmesartan medoxomil through a developed and validated HPLC method," RSC Advances, vol. 3, no. 27, pp. 10887-10893, 2013.

[25] N. A. Alhakamy, A. U. Fahmy, S. M. Badr-Eldin et al., "Optimized icariin phytosomes exhibit enhanced cytotoxicity and apoptosis-inducing activities in ovarian cancer cells," Pharmaceutics, vol. 12, no. 4, p. 346, 2020.

[26] S. A. Kumbhar, C. R. Kokare, B. Shrivastava, B. Gorain, and H. Choudhury, "Antipsychotic potential and safety profile of TPGS-based mucoadhesive aripiprazole nanoemulsion: development and optimization for nose-to-brain delivery," Journal of Pharmaceutical Sciences, vol. 110, no. 4, pp. 1761-1778, 2021.

[27] D. G. Gadhave, A. A. Tagalpallewar, and C. R. Kokare, "Agranulocytosis-protective olanzapine-loaded nanostructured lipid carriers engineered for CNS delivery: optimization and hematological toxicity studies," AAPS PharmSciTech, vol. 20, no. 1, 2019.

[28] A. Hussain, A. Samad, S. K. Singh et al., "Nanoemulsion gelbased topical delivery of an antifungal drug:in vitroactivity andin vivoevaluation," Drug Delivery, vol. 23, no. 2, pp. 642-657, 2016.

[29] U. S. Kadimi, D. R. Balasubramanian, U. R. Ganni, M. Balaraman, and V. Govindarajulu, "In vitro studies on liposomal amphotericin B obtained by supercritical carbon dioxide-mediated process," Nanomedicine: Nanotechnology, Biology and Medicine, vol. 3, no. 4, pp. 273-280, 2007.

[30] A. Malasiya and A. Goyal, "Method development and validation of RP HPLC method for assay and related substances of luliconazole in topical dosage form," International Journal of Pharmaceutical Chemistry and Analysis, vol. 4, no. 2, p. 50, 2017.

[31] P. V. Pople and K. K. Singh, "Development and evaluation of topical formulation containing solid lipid nanoparticles of vitamin A," AAPS PharmSciTech, vol. 7, no. 4, pp. E63-E69, 2006.

[32] H. Choudhury, B. Gorain, B. Chatterjee, U. K. Mandal, P. Sengupta, and R. K. Tekade, "Pharmacokinetic and pharmacodynamic features of nanoemulsion following oral, intravenous, topical and nasal route," Current Pharmaceutical Design, vol. 23, no. 17, pp. 2504-2531, 2017.

[33] B. Gorain, H. Choudhury, A. Kundu et al., "Nanoemulsion strategy for olmesartan medoxomil improves oral absorption and extended antihypertensive activity in hypertensive rats," Colloids and Surfaces B: Biointerfaces, vol. 115, pp. 286-294, 2014.

[34] H. Choudhury, N. F. B. Zakaria, P. A. B. Tilang et al., "Formulation development and evaluation of rotigotine mucoadhesive nanoemulsion for intranasal delivery," Journal of Drug Delivery Science and Technology, vol. 54, p. 101301, 2019.

[35] S. Md, N. A. Alhakamy, H. M. Aldawsari et al., "Formulation design, statistical optimization, and in vitro evaluation of a naringenin nanoemulsion to enhance apoptotic activity in A549 lung cancer cells," Pharmaceuticals, vol. 13, no. 7, p. 152, 2020.

[36] X. Li, L. Wang, and B. Wang, "Optimization of encapsulation efficiency and average particle size of Hohenbuehelia serotina polysaccharides nanoemulsions using response surface methodology," Food Chemistry, vol. 229, pp. 479-486, 2017.

[37] N. H. Che Marzuki, R. A. Wahab, and M. Abdul Hamid, "An overview of nanoemulsion: concepts of development and 
cosmeceutical applications," Biotechnology \& Biotechnological Equipment, vol. 33, no. 1, pp. 779-797, 2019.

[38] M. Pandey, H. Choudhury, O. C. Yeun et al., "Perspectives of nanoemulsion strategies in the improvement of oral, parenteral and transdermal chemotherapy," Current Pharmaceutical Biotechnology, vol. 19, no. 4, pp. 276-292, 2018.

[39] S. Migliozzi, G. Meridiano, P. Angeli, and L. Mazzei, "Investigation of the swollen state of Carbopol molecules in nonaqueous solvents through rheological characterization," Soft Matter, vol. 16, no. 42, pp. 9799-9815, 2020.

[40] A. Islam, M. Riaz, and T. Yasin, "Structural and viscoelastic properties of chitosan-based hydrogel and its drug delivery application," International Journal of Biological Macromolecules, vol. 59, pp. 119-124, 2013.

[41] A. Simões, M. Miranda, C. Cardoso, F. Veiga, and C. Vitorino, "Rheology by design: a regulatory tutorial for analytical method validation," Pharmaceutics, vol. 12, no. 9, pp. 820-827, 2020.

[42] H. Kansagra and S. Mallick, "Microemulsion-based antifungal gel of luliconazole for dermatophyte infections: formulation, characterization and efficacy studies," Journal of Pharmaceutical Investigation, vol. 46, no. 1, pp. 21-28, 2016.

[43] D. A. Godwin, B. B. Michniak, and K. E. Creek, "Evaluation of transdermal penetration enhancers using a novel skin alternative," Journal of Pharmaceutical Sciences, vol. 86, no. 9, pp. 1001-1005, 1997.

[44] S.-C. Shin and J.-S. Choi, "Enhanced efficacy of triprolidine by transdermal application of the EVA matrix system in rabbits and rats," European Journal of Pharmaceutics and Biopharmaceutics, vol. 61, no. 1-2, pp. 14-19, 2005.

[45] Y. Zhang, M. E. Lane, and D. J. Moore, "An investigation of the influence of PEG 400 and PEG-6-caprylic/capric glycerides on dermal delivery of niacinamide," Polymers, vol. 12, no. 12, pp. 2907-2911, 2020. 